\title{
A Review of the Scientific Evidence for Acupuncture and Dry Needling Compared in Common Sporting Conditions and Medical Disorders
}

\author{
Alex Huntly, Daniel Berdejo-del-Fresno* \\ England Futsal National Team, The Football Association, London, UK \\ *Corresponding author: daniberdejo@gmail.com
}

Received October 15, 2014; Revised October 17, 2014; Accepted October 20, 2014

\begin{abstract}
There is an evidence base of varying strength for the use of acupuncture in conditions, such as chronic low back pain, myofascial trigger point, osteoarthritis, pain relief, performance enhancement and respiratory disorders. The aim of this study was to review the evidence base of acupuncture for common conditions that occur in sport. A literature review of how acupuncture works and what it can treat was performed. Evidence for each condition was critically appraised. There are significant differences in the perceived effectiveness of acupuncture for different conditions and mechanisms. Chronic low back pain, myofascial trigger point, acute low back pain and osteoarthritis were perceived to be effective. All of the reviewed mechanisms of acupuncture were perceived more effective than Chinese meridian. Those conditions that were rated as less effective generally had less supporting evidence or were not relevant to sporting populations.
\end{abstract}

Keywords: trigger point, low back pain, traditional chinese medicine

Cite This Article: Alex Huntly, and Daniel Berdejo-del-Fresno, "A Review of the Scientific Evidence for Acupuncture and Dry Needling Compared in Common Sporting Conditions and Medical Disorders." American Journal of Sports Science and Medicine, vol. 2, no. 5 (2014): 181-189. doi: 10.12691/ajssm-2-5-2.

\section{Introduction}

This article takes a critical look at the role of acupuncture for treating sports people from a clinician's perspective. This is timely as attitudes of health professionals strongly indicate an interest (Rampes et al., 1997) and there has been a recent flurry in acupuncture research (White at al., 2008; Longbottom, 2010). The conclusions of this report are not limited to one particular health profession but any person that may administer treatment to an active person, such as a doctor, physiotherapist or other allied health care professional.

\subsection{Acupuncture History}

Acupuncture is thought to have originated in ancient China and involves the insertion of fine needles into various locations of the body that illicit a strong and largely predictable reaction. In Traditional Chinese acupuncture these points are believed to correspond to meridians of energy that are unblocked by the needling process. There are conflicting theories as to the time of origin but it could have been as far back as 3200BC (Dorfer et al., 1998). Traditional acupuncture has been used to treat a plethora of conditions including pain, systemic disorders, psychological disorders and addiction (White, 2006). These theories have remained unchanged since the 16th and 17th centuries (Hempen \& Chow,
2006). More recently from the 1950s onwards acupuncture has been the subject of scientific trials and an area of great interest for researchers. The work of these pioneers attempts to explain acupuncture in the context of our current scientific understanding of pain and healing mechanisms. This is the basis of Western medical acupuncture. As a result both Traditional Chinese medicine and Western medical acupuncture exist as separate entities. Both have trained individuals and are valid forms of treatment. To be integrated into health care there must be cost analysis and good quality evidence for its use to be accepted by the medical community.

\subsection{East vs West}

Amongst health professionals and in the research there are many variations in the practice of acupuncture and often two approaches are cited 'conventional medicine' and 'Traditional Chinese acupuncture'. In actual fact there are many forms of approach to acupuncture and therefore this simplistic view does not accommodate the variety of practice that occurs. The rationale behind each treatment method is very different however both agree that sensitivity to a patients symptoms and tailoring treatment dose and type to the individual are important to success. Of course there are variations in the way practice is performed and clinicians will justify their intervention based on personal experience. Having a sound understanding of how a positive outcome is achieved will clarify clinical reasoning and benefit medicine as a whole. 


\subsection{Definitions}

There is great variation and confusion about the fundamental definition of acupuncture. This depends on the clinicians experience, background, teaching, individual understanding and skill. For the purposes of this study the definition of acupuncture is: "Any therapy involving the insertion of one or more needles to achieve a treatment effect". This may include manual insertion of needles, electro acupuncture and auricular acupuncture. Acupressure is not considered in this review as it is a manual therapy and is practiced without needles. In this text the terms scientific, medical and Western medical acupuncture are used interchangeably. Where no explanation is given these phrases will mean acupuncture described below.

\subsection{Western Medical Acupuncture}

Western medical acupuncture is an approach that 'interprets acupuncture according to current understanding of the body's structure and function' (White et al., 2008, pg 7).

This is the essential difference between Western medical acupuncture and Traditional Chinese Medicine. This critical view is taken throughout this study in an attempt to make sensible conclusions that are backed up by hard science and cost effective in nature. A typical Western medical acupuncture treatment will consist of a routine clinical assessment and examination. From these findings the health professional uses clinical reasoning to diagnose the patient and formulate a treatment plan. This intervention may or may not involve the use of acupuncture applied by the health professional. The decision to use acupuncture will be based on the known effect of acupuncture to influence the patient's condition. This mechanistic view is typical of Western medical acupuncture for example reducing pain in a dermatome via stimulation of nerves in the skin around the area of pain. Therefore much of the literature focuses upon mechanisms by which acupuncture effects the body. Many of its actions influence the nervous system and will be discussed in detail below.

\subsection{Acupuncture and Sport}

Clinical reasoning should be as rigorous in sport as it is in all other forms of health care. Clear understanding of the mechanism by which acupuncture works will improve outcomes for athletes. If there is an application for treating muscle tension and pain with acupuncture a large group of sports people would benefit from this particular intervention. Any patient who is active may be considered within this study and may range from a recreational noncompetitive sports person to an elite athlete.

\subsection{Theoretical Background of Acupuncture}

A Western acupuncturist makes a medical diagnosis according to conventional methods and uses needles to influence the physiology of the body according to a 'known' response' (White et al., 2008). A known response is based on a proven theory or clinical experience. This intervention will be part of a range of medical methods. For the purposes of this review the mechanisms of acupuncture have been divided into 5 proposed mechanisms drawn from White et al. (2008) outlined below.

Local effects: Needling produces several effects in the skin that have a local effect. Firstly stimulation of sensory nerves produces action potentials. As a result, local chemicals are released one of which is called calcitonin gene related peptide (CGRP). This is known to cause blood vessel dilation so that blood flow increases around the site of needling (Sato et al., 2000). This increase in blood flow locally is thought to improve healing (White et al., 2008).

Segmental analgesia: Nocioceptive signals elicited by acupuncture travel via afferent fibres to the spinal cord where they depress activity in the dorsal horn 'closing the pain gate' in the way that was first described by Wall et al. (2006). This effect is used therapeutically in a number of different situations and is well established particularly in the case of electro-acupuncture.

Extra-segmental analgesia: The action potentials produced by needling then travel up from the spinal cord to the brainstem where they stimulate the body's own pain suppressing mechanisms at the peri aqueductal grey matter (PAG) this is also known as "descending inhibition" (White et al., 2008). This analgesic effect is not restricted to any single segment but is achieved by a certain amount of stimulation in the nervous system. Neuromodulators are released as a result of acupuncture and modify neural function via endogenous opioids such as endorphins and enkephalins (White et al., 2008; Filshie \& White, 1998).

Central effects (brain): Once action potentials have reached the brain several structures are stimulated. These include the cerebral cortex, the hypothalamus and limbic system. These areas regulate the emotional affective component of pain 'how a person feels about their pain'. These brain areas have various pain regulation effects which will be discussed later. Needling may also influence the autonomic nervous system and various hormones (White et al., 2008).

Myofascial trigger points: Myofascial trigger points are small knots of tight muscle fibres. They can be caused by poor posture or overloading of a muscle (Simons et al., 1999). These are often identified because they refer pain in a predictable fashion. There is no formal investigation for diagnosing myofascial trigger points but they can be identified clinically by palpitating the tense, tender area of muscle and reproducing pain (White et al., 2008; Simons et al., 1999).

Other mechanisms: Pain mechanisms are not fully understood and more research using functional MRIs will improve our knowledge of the functions of certain brain areas influenced by acupuncture. Other areas include the action of needling on the connective tissue (Langevin, 2006; Langevin \& Yandow, 2002), neurotransmitters (White et al., 2008) and the effect of acupuncture on gene expression of neuropeptides (Guo et al., 1996; Gao et al., 1997). Other interpretations of Western medical acupuncture by eminent practitioners focus more on clinical examination and experience of the practitioner. Mann (2000) for example does not subscribe to the meridian system and proposes that areas rather than points should be needled. Mann is also the first to describe periosteal pecking as a treatment which involves needling of the periosteum to achieve strong acupuncture stimulation. 


\section{Literature Review}

\subsection{Mechanisms of Action}

\subsubsection{Local Effects}

The first experiments on the local effects of acupuncture were conducted by the Chinese. One such article by Chung and colleagues in 1973 demonstrated that acupuncture needles had no effect if they were inserted into an area that had been anaesthetized by injection of local anaesthetic, proving for the first time that needling largely affects the nervous system. Wang and colleagues (1985) followed up this work in 1985 by demonstrating that acupuncture generates nerve action potentials leading away from the treatment area.

\subsection{1.a. Sensory nerves in the skin and muscle}

There are several types of sensory fibres within the skin and muscle, see Figure 1.

\begin{tabular}{|c|c|c|c|c|}
\hline Fibre type & $\begin{array}{c}\text { In } \\
\text { Skin }\end{array}$ & $\begin{array}{c}\text { In } \\
\text { Muscle }\end{array}$ & Ending & Sensation \\
\hline Large myelinated & - & I & Muscle spindle & None \\
\hline Large myelinated & A $\beta$ & II & Encapsulated and free endings & Light touch, pressure, vibration \\
\hline $\begin{array}{c}\text { Medium } \\
\text { myelinated }\end{array}$ & A $\gamma$ & II & $\begin{array}{c}\text { Muscle spindle secondary, encapsulated } \\
\text { endings }\end{array}$ & \begin{tabular}{c} 
Numbness \\
\hline Small myelinated
\end{tabular} \\
A $\delta$ & II & Free endings & cold \\
\hline $\begin{array}{c}\text { Small } \\
\text { unmyelinated }\end{array}$ & C & IV & Free endings & Soreness aching, itch heat; calmness \\
\hline
\end{tabular}

Figure 1. Table showing the nerve fibre type adapted from (White et al., 2008)

Essentially acupuncture stimulates $\mathrm{A} \delta$ and type II and III afferent muscle fibres (White et al., 2008). An afferent fibre travels from distal receptors to the central nervous system (Saladin \& Van Wynsberghe, 2001). When these nerve fibres are successfully stimulated the patient will experience a range of sensations. This fall into two categories acute pain and a sensation know as de $q i$ (MacPherson \& Asghar, 2006). De qi is widely discussed in the literature and often cited as being associated with successful needling technique. It feels like a 'dull ache' and is a very recognisable sensation to acupuncturists and adequately informed patients. Nerve conduction studies have shown that the onset of de $q i$ is accompanied by action potentials that are characteristically typical of $\mathrm{A} \delta$ fibre stimulation (Wang et al., 1985). Further work by Andersson \& Lundeberg (1995) has hypothesised that the sensation of de qi is comparable to deep muscle ache after exercise that arises from stimulation of free nerve endings of type II/III fibres in the muscle. The importance of the strength and type of sensation has yet to be established but many agree that de qi is important to the success of an intervention (White et al., 2006).

\subsection{1.b. Neuropeptides}

Upon inserting a needle, free nerve endings are stimulated. This occurs in a web or reticulum of nerve fibres and is known as an axon reflex (White et al., 2008). Upon stimulation they release several vasodilatory neuropeptides into the muscle and skin they innervate, one of which is calcitonin gene related peptide (CGRP) (White, 2006). Other neuropeptides that are known to be released include vasointestinal active peptide, neuropeptide $\mathrm{Y}$ and substance P (White et al., 2008; Weidner et al., 2000). The result is an increase of blood flow to the area which, among others functions, allows for nutrition of the local structure. This can be observed as redness around the needle site. Sensory neuropeptides also regulate immune responses and assist healing responses (Brain, 1997). This is supported by Sandberg and colleagues (2003) who studied blood flow after needling in healthy volunteers and showed that there is a stepwise increase in local blood flow when the needle is inserted to the skin, reaches the depth of muscle and de qi respectively. Other observed, but less well understood, effects include increased activity of local glands such as the salivary glands from local needling (Blom et al., 1993) and remodelling of connective tissue (Langevin et al., 2006).

Acupuncture points are an area of controversy and disagreement. A detailed discussion is beyond the scope of this text but it is generally agreed that there are certain 'areas' usually in muscles that elicit a strong response from deep needling. Certain points are generally regarded as useful in treating certain problems (White et al., 2008).

Strength of stimulation or 'dosage' is also an area of debate. There is no strong empirical data to suggest: what number of needles to use; depth; and amount of stimulation. However convention suggests that de $q i$ should be obtained and somewhere between 1 and 20 needles can be used (White et al., 2008).

\subsubsection{Segmental Aanalgesia}

Segmental analgesia is a theoretical mechanism of action by acupuncture and is supported by clinical experience and some good quality trials. It is achieved by needling in the same myotome or dermatome as the nocioceptive fibres that are causing pain (White et al., 2008; Filshie \& White, 1998; Ernst \& White, 1999). Any acupuncture point that shares an innovation via that spinal segment can be chosen (Longbottom, 2010). Clinical experience in humans shows the insertion of a needle into a tender area resets the threshold to a more normal value and produces lasting pain relief (Filshie \& White, 1998). This is supported by the work of Frost et al. (1980) who injected tender regions in two groups of patients and showed that needling (with injection of saline) and needling with injection of mepvacaine after three sessions two or three days apart resulted in relief just by the action of needling alone. Garvey et al. (1989) in a randomised double-blind study demonstrated a similar improvement of acupuncture on low back pain (63\%) when compared with injection of lidocaine or a lidocaine containing steroid (42\%). Macdonald et al. (1983) also found a significantly superior result of segmental acupuncture versus placebo (inactive TENS) in a single-blind randomised study. Despite these positive results, little is 
known about the underlying analgesic mechanism of acupuncture (Filshie \& White, 1998). In a review on the subject Treede et al. (1992) could not conclusively offer a mechanism of action for segmental analgesia but it is likely to be the combination of pain gate at the dorsal horn and the effects of acupuncture on the higher centres of the brain (Filshie \& White, 1998).

\subsubsection{Extra Segmental Effects}

There are two areas in acupuncture that have demonstrated extra segmental effects, these are: neuromodulators (a substance, other than a neurotransmitter, released by a neuron and transmitting information to other neurons, altering their activities (White et al., 2008)); and descending inhibitory pain control via action of the PAG, blocking pain signals in the dorsal horn from the brain stem down (White et al., 2008). Neuromodulators became popular after Chen et al. (1983) studied the effect of needling rabbits and demonstrated that the opioids in the cerebrospinal fluid changed along with an increase in the pain threshold. The effect of neuromodulators differs according to each individual but clearly has a role in improving pain thresholds in humans (White et al., 2008). Electro acupuncture and transcutaneous electrical stimulation (TENS) work by the same mechanism and different intensities and frequencies stimulate different nerves and release different neuromodulators (Filshie \& White, 1998).

\subsubsection{Central Effects}

Whilst local effects are logical in treating local symptoms, needling far away from the symptomatic area is a common technique used in acupuncture (White et al., 2008). There is strong anecdotal evidence and more recently functional Magnetic Resonance Imagining (fMRI) evidence for this phenomenon, although the area is far from fully described. Central effects include calmness and a good night sleep (White et al., 2008). Central effects are particularly useful in chronic conditions as they act on the affective aspect of pain. Patients may still feel the pain but it bothers them less (White et al., 2008). This phenomenon is thought to be the result of actions by the limbic system. The limbic system processes and responds to pain (White et al., 2008). It is interconnected to a group of structures deep within the brain; these include the amygdale, hippocampus, parahippocampus, anterior cingulated cortex, prefrontal cortex, septum, nucleus accumbens, hypothalamus, insula and caudate (White et al., 2008). There is now considerable evidence that acupuncture has a considerable effect on the limbic system (Hui et al., 2000; Hui et al., 2005; Pariente et al., 2005). This general effect of acupuncture almost certainly does not depend on the needle site and has an emotional component (White et al., 2008). This is supported by sham controls where the patient is convinced of needle insertion when in fact a blunt pin is stimulating the skin ( $\mathrm{C}$ sensory fibres) and marked activation is observed in the limbic system (Lund \& Lundeberg, 2006).

Though our knowledge of the exact mechanism is still limited, the evidence is accumulating and at least in certain conditions acupuncture can have a beneficial effect on patients. One interpretation is that the affective component of pain will respond to any form of acupuncture that stimulates the limbic system, while the sensory component of pain is likely to respond better if the specific mechanism of segmental analgesia is elicited. The limbic system can be stimulated by non specific needling (and even sham acupuncture) whereas segmental acupuncture relies on de $q i$ in an appropriate spinal segment (White et al., 2008). This hypothesis goes some way to explaining the findings of the large insurance company trial of acupuncture in Germany in the early 2000s (Haake et al., 2007; Molsberger et al., 2006; Linde et al., 2005; Brinkhaus et al., 2003). They found that sham acupuncture and acupuncture were both equally effective at treating tension headache, back pain and migraine. However acupuncture (segmental) was significantly superior to sham for knee pain due to the required segmental effect of the sensory component of knee pain cause by joint degeneration. The theory proposed is that tension headache, back pain and migraine have a high affective component which responds to non specific needle stimulation (Lund \& Lundeberg, 2006). The affective component of pain is rarely measured in trials (White et al., 2008) but one study has done this. Thomas et al. (1991) took forty-four patients with chronic cervical osteoarthritis and treated with acupuncture, shamacupuncture, diazepam or placebo-diazepam. Pain was rated on visual analogue scales before, during, and after treatment. Two scales were separately used to rate the intensity (sensory component) and the unpleasantness (affective component) of pain. The results from this trial were that diazepam, placebo-diazepam, acupuncture and sham-acupuncture have a more pronounced effect on the affective than on the sensory component of pain.

\subsubsection{Myofascial Trigger points}

A myofascial trigger point (MTrP) as defined by Simons et al. (1999) is "a hyper irritable spot in skeletal muscle that is associated with a hyper sensitive palpable nodule in a taut band. The spot is painful on compression and can give rise to characteristic referred pain, referred tenderness, motor dysfunction, and autonomic phenomena."

The existence of MTrPs was an area of controversy but in recent years it has become widely accepted and researched although it is still an area which some do not commonly diagnose and treat (Simons et al., 1999). Interestingly the location of trigger points often correlate precisely with that of acupuncture points (Melzack et al., 1997). Some hypothesise that it may be the desire to treat MTrPs that lead to the invention of acupuncture (Ernst \& White, 1999). Precipitating factors include mechanical stress, nutritional inadequacies, metabolic and endocrine inadequacies, psychological factors, chronic infection, impaired sleep, radiculopathy and chronic visceral disease (Simons et al., 1999).

Diagnosis is of a clinical nature made using the definition above. There is yet to be an adequate test (Simons et al., 1999), however training and experience in diagnosis improves interrater reliability (Njoo \& Vanderboes, 1994; Gerwin et al., 1997). Electromyography can help to confirm the presence of a MTrP in the presence of low voltage motor end plate noise (Simons et al., 1999).

MTrPs can be deactivated in a number of ways. These include direct sustained pressure (Brukner \& Khan, 2007), stretching (Brukner \& Khan, 2007), ice spray and 
stretching (Simons et al., 1999), massage (Simons et al., 1999), transcutaneous electrical nerve stimulation and needling (Lomgbottom, 2010; Simons et al., 1999; Brukner \& Khan, 2007). It is generally regarded that needling is best for chronic trigger points so long as the muscle is accessible and the practitioner is adequately trained (Simons et al., 1999). In fact White et al. (2008) states that acupuncture is the most rapidly effective treatment for trigger points. This is likely because manual methods can require more treatment sessions (Simons et al., 1999). Some practitioners inject a local anaesthetic during needling but Jaeger et al. (1987) in a double blind controlled trial demonstrated that needling alone is as effective. Hong (1994) found similar results and showed that a local twitch response must be elicited otherwise needling with or without anaesthetic is likely to be ineffective. A local twitch response is a reflex contraction within a tense muscle that traverses a trigger point (Simons et al., 1999). Ceccherelli et al. (2001) in a related randomised controlled study $(n=44)$ demonstrated that deep acupuncture is significantly more effective at treating shoulder pain from trigger points than superficial at the time of treatment, at one month and three months followup. This work however did not mention twitch response.

The number of sites that need to be injected per visit and the number of visits required depend on the patient's condition and the practitioner's skill and judgement (Simons et al., 1999). Exercises should be given after needling to maximise the muscle lengthening effect of successful treatment of a trigger point (Simons et al., 1999).

Clinical trials are lacking but one study by Edwards \& Knowles (2003) examined patients who were referred for physiotherapy and selected 40 respondents with trigger points diagnosed by point tenderness, pain recognition and limited movement of the muscle. One group had superficial needling and were taught stretches, another group was taught stretches only, and the final group had no intervention. There was a trend in favour of acupuncture at follow-up, but the difference was only significant $(\mathrm{P}=0.043)$ at three weeks after treatment. A larger sample size may demonstrate a bigger treatment effect by eliminating type II error. One trial by Huguenin et al. (2005) investigated the effects of dry needling in the gluteal muscles on straight leg raise in athletes who suffer from posterior thigh pain. The results were that range of motion around the hip was not significantly different however activity related muscle pain and tightness was significantly improved by dry needling. A meta-analysis (Tough et al., 2009) reviewing acupuncture for MTrPs concluded deep needling directly into MTrPs is more effective than no treatment and has a trend towards being superior than sham needling into the muscle at a different area although this is not statistically significant. More studies are needed to compare acupuncture with other treatments to inform future clinical guidelines for treating MTrPs (Fleckenstein et al., 2010).

\subsection{Acupuncture for Sporting Conditions}

So far the mechanistic actions of acupuncture have been discussed in detail. Now each specific condition will be examined. Conditions have been selected from the World Health Organisations (WHO) Acupuncture review and analysis of reports and clinical trials (2003).
Low back pain: Low back pain is commonly treated with acupuncture and endorsed by the National Institute of Clinical Excellence (NICE) as a treatment option instead of exercise or manual therapy (NICE, 2009). Mannheimer et al. (2005) performed a meta-analysis of 33 randomised controlled trials on this topic. Back pain was divided into two subgroups: acute and chronic low back pain. The recommendations of this study were that chronic pain was alleviated by acupuncture in the short term and acupuncture is significantly more effective than sham treatment and waiting list controls. There is not enough data to prove or disprove its superiority over other forms of treatment and more studies are required to establish longer term results for pain. For acute low back pain data were lacking to support or reject the use of acupuncture. Furlan et al. (2005) conducted a Cochrane review on the same topic and came to the same conclusions. After those reviews Thomas et al. (2005) compared acupuncture with GP care and acupuncture showed a trend towards being more effective although it was only significantly superior than GP care in one outcome measure of pain.

Tendinopathy: There are two positive studies for the use of acupuncture for tennis elbow. Haker et al. (1990) found deep needling at acupuncture points was more effective than superficial needling the same points. Fink et al. (2002) found 10 treatments twice per week with genuine acupuncture were superior to sham needling. Trinh et al. (2004) reviewed six high quality studies and concluded there is a strong case for the use of acupuncture in the short term on tennis elbow, this is because tennis elbow is self limiting after 12 months (Smidt et al., 2002).

Osteoarthritis: Pain in the knee from osteoarthritis has been investigated by White et al. (2007). In this systematic review 13 randomised controlled trials were included. To qualify for inclusion into this study they had to have at least six treatments, at least once treatment per week, at least four points needled for at least 20 minutes with either manual stimulation or electrical stimulation. In addition sham controls could only be called true sham if they did not needle the legs in the same spinal segments as the knee joint. The meta-analysis clearly showed that acupuncture was superior to sham for both pain and function both at the time of intervention and at long term follow up (6-12 months).

Performance enhancement: A few small studies have attempted to measure acupuncture for performance enhancement in cycling (Ozerkan et al., 2009; Dhillon, 2008). None have demonstrated statistically significant improvements above trend and do not propose a mechanism of action for why acupuncture would improve performance.

\subsection{Acupuncture for other Conditions}

Blood disorders: Four Chinese articles in the WHO acupuncture review (2003) advocate acupuncture for treating various blood disorders. This includes treating leukopenia (low white blood cell count) due to chemotherapy and benzene intoxication. A search of Medline revealed no English language papers to support this.

Cardiovascular disease: For cardiovascular disease in the WHO Acupuncture review (2003) there are 18 papers of which 17 were not obtainable in English language. That 
one English language trial showed evidence that acupuncture can improve cardiovascular function in patients with mild angina pectoralis (AP) (Ballegaard et al., 1990) and another by the same author showed that this effect occurs in patients with severe AP (Ballegaard et al., 1986). However evidence for acupuncture in the long term management of hypertension has not demonstrated a significant role (White et al., 2008; Kim \& Zhu, 2010; Lee et al., 2009; Mukaino et al., 2005).

Depression: Despite promising hopes for acupuncture treating depression the evidence has yet to materialise (Mukaino et al., 2005) a Cochrane review cites a high risk of positive publication bias in acupuncture trials as the main reason for the failure to make evidence based conclusion (Smith et al., 2010).

Digestive disorders: 11 trials are cited in the WHO acupuncture review (2003) non were obtainable through Ovid Medline.

Hay fever: One randomised control trial has shown that acupuncture for treating hay fever has a trend towards beneficial outcomes for patients (Williamson et al., 1996) this promising result and other anecdotal evidence especially in the Traditional Chinese Medicine approach has convinced many acupuncturists that it is effective for treating this condition. However there is a lack of statistically significant trials.

Infection: There are no English language articles for the use of acupuncture for treating infection. However there are 6 Chinese papers advocating its use for various infections by increasing immune function were referenced in the WHO acupuncture review (2003, pg 12).

Migraine: A review of treatment of migraine using acupuncture by Melchart et al. (2001) showed a trend towards acupuncture being superior to sham but the overall quality of the studies was too weak to draw strong conclusions. Since that review a large RCT compared acupuncture with usual care (Vickers et al., 2004). The acupuncture patients experienced significantly fewer days headache (22 days less at 1 year follow up) than the control group. This shows that acupuncture has persisting, clinically relevant benefits for primary care patients with migraine.

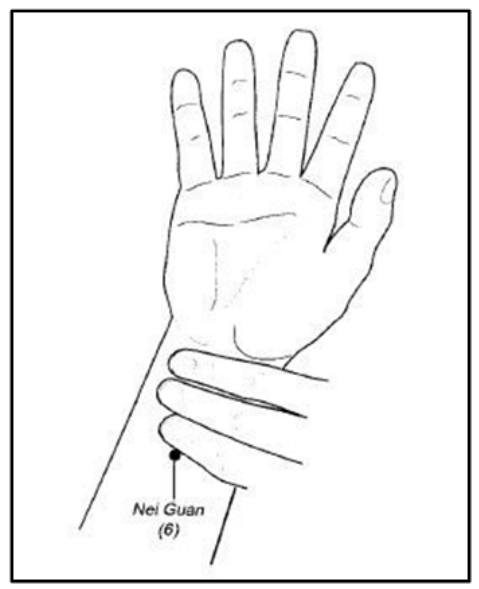

Figure 2. PC6 acupuncture point

Nausea: Various forms of acupuncture have been shown efficacious for treating nausea: for example a Cochrane review concluded that stimulation of the acupuncture point PC 6 (see Figure 2) significantly reduces postoperative nausea (Lee \& Done, 2004); it is also efficacious in treating nausea in early pregnancy (Smith et al., 2002); and it has also been shown to reduce vomiting after chemotherapy (Ezzo et al., 2005).

Neurological disorders: Acupuncture has been suggested for a wide variety of neurological conditions from neuralgia to stroke (WHO, 2003). However there are no good quality RCTs supporting its use in epilepsy, Alzheimer's disease, Parkinson's disease, ataxic disorders, multiplesclerosis, amyotrophic lateral sclerosis, spinal cord injury, and stroke rehabilitation (Lee et al., 2007).

\section{Obstetric and Gynaecological disorders:} Acupuncture has been suggested for treating a range of obstetric disorders listed below;

Infertility: The research on infertility has suggested that acupuncture may have a number of beneficial actions though the evidence is not conclusive (Stener-Victorin \& Humaidan, 2006). Some trials have shown that in women who have anovulation (unable to ovulate) due to polycystic ovarian syndrome have long lasting improvements with electro acupuncture (White et al., 2008). Also a systematic review by Mannheimer et al. (2008) concluded current preliminary evidence suggests that acupuncture given with embryo transfer improves rates of pregnancy and live birth among women undergoing in vitro fertilisation (IVF).

Labour pain: acupuncture seems to be useful in reducing labour pain according to 3 trials summarised in a recent review (Lee \& Ernst, 2004). Further more women who chose acupuncture are less likely to require epidural (Lee \& Ernst, 2004).

Premenstrual syndrome (PMS): Cho et al. (2010a) conducted a review that included trials that showed acupuncture may be beneficial to patients with PMS. However there is insufficient evidence to support this conclusion due to methodological flaws in the studies, concealment of allocation, blinding and outcome measures.

Dysmenorrhea: is a condition characterised by severe uterine pain during menstruation that can affect female athletes (Brukner \& Khan, 2010). Cho et al. (2010b) in a recent review on the acupuncture for dysmenorrhea concluded that the evidence is not convincing. Proctor et al. (2002) also showed a negative trend.

Respiratory conditions: Exercise induced asthma is a condition that affects a significant number of athletes (Brukner \& Khan, 2010). The standard care is short acting bronchodilators and long acting anti-inflammatory inhalers with progression to further pharmacological interventions if symptoms worsen. While acupuncture cannot treat asthma (WHO, 2003) there are some trials that indicate it may attenuate symptoms alongside usual medication (Fung et al., 1986; Yu \& Lee, 1976). However the sample sizes of these trials were small ( $n=19$ and $n=20$, respectively) and the latter trial was uncontrolled. Other studies do not find these results (Biernacki \& Peake, 1998) but do record a beneficial result by reduction in bronchodilator medication use and an improvement in quality of life.

Skin diseases: In some countries acupuncture is favoured for treating skin diseases such as acne (WHO, 2003). Five trials were identified in the WHO acupuncture review (2003) but only one was English language (Lundeberg et al., 1987). This preliminary trial showed significant results and concluded that acupuncture has 
potential as a treatment for pruritus (a painful itchy skin condition).

Urogenital disorders: Two papers are cited in the WHO acupuncture review (2003) both were not obtainable. A search of Ovid Medline identified one pilot study investigating the feasibility of acupuncture for treating incontinence. This study demonstrated that acupuncture can exert a beneficial response and concluded that there more investigation is indicated (WHO, 2003; Engberg et al., 2009).

\section{Summary}

For many conditions there is not enough evidence to support or refute the use of acupuncture. However in some conditions such as chronic low back pain and chronic knee pain (due to osteoarthritis) there is compelling evidence for the use of acupuncture. In some instances the use of sham interventions is as effective as real acupuncture especially in conditions that have a highly affective pain component (migraine, tension headaches); in these instances acupuncture is better than waiting list control groups.

Finally, since the quality of acupuncture trials is in general remarkably poor, two papers have developed indexes to consider the adequacy of the technique of the acupuncture treatment the placebo technique. Basically, these articles may guide you when analyzing acupuncture trials (Gonçalves-Nordon et al., 2012; Gonçalves-Nordon et al., 2013). Also, the same author (Gonçalves-Nordon, 2013) studied the relation between the quality of the article and the effectiveness of acupuncture; in summary, the better the study is performed, the higher the effectiveness of acupuncture.

\section{References}

[1] The peripheral afferent pathway in acupuncture analgesia. ChungHua i Hsueh Tsa Chih [Chinese Medical Journal]. 1974; 6: 360364.

[2] Andersson S, Lundeberg T. Acupuncture - from empiricism to science - functional backgroud to acupuncture effects in pain and disease. Medical Hypotheses. 1995; 45 (3): 271-281.

[3] Ballegaard S, Jensen G, Pedersen F, Nissen VH. Acupuncture in severe, stable angina-pectoris - A randomised trial. Acta Medica Scandinavica. 1986; 220 (4): 307-313.

[4] Ballegaard S, Pedersen F, Pietersen A, Nissen VH, Olsen NV. Effects of acupuncture in moderate, stable angina-pectoris - a controlled study. Journal of Internal Medicine. 1990; 227 (1): 2530.

[5] Biernacki W, Peake MD. Acupuncture in treatment of stable asthma. Respiratory Medicine. Sep 1998; 92 (9): 1143-1145.

[6] Blom M, Lundeberg T, Dawidson I, Angmarmansson B. Effects on local blood flux of acupuncture stimulation used to treat xerostomia in patients suffering from sjogrens-syndrome. Journal of Oral Rehabilitation. 1993; 20 (5): 541-548.

[7] Brain SD. Sensory neuropeptides: their role in inflammation and wound healing. Immunopharmacology. 1997; 37 (2-3): 133-152.

[8] Brinkhaus B, Becker-Witt C, Jena S, et al. Acupuncture randomized trials (ART) in patients with chronic low back pain and osteoarthritis of the knee - Design and protocols. Forschende Komplementarmedizin Und Klassische Naturheilkunde. 2003; 10 (4): 185-191.

[9] Brukner P, Khan K. Clinical sports medicine. 3rd ed. London: McGraw-Hill; 2007.

[10] Ceccherelli F, Bordin M, Gagliardi G, Caravello M. Comparison between superficial and deep acupuncture in the treatment of the shoulder's myofascial pain: A randomized and controlled study.
Acupuncture \& Electro-Therapeutics Research. 2001; 26 (4): 229238.

[11] Cook J. Funky treatments in elite sports people: do they just buy rehabilitation time? British Journal of Sports Medicine. 2010; 44 (4): 221-221.

[12] Chen B, Wang D, Pan I. Changes of opiate-like substance level in the perfusate of peri aqueductal gray after electro acupuncture and brain stimulation in rabbit. Acta Physiologica Sinica. 1983; 34 (4): 385-391.

[13] Cho SH, Hwang EW. Acupuncture for primary dysmenorrhoea: a systematic review. BJOG: An International Journal of Obstetrics \& Gynaecology. Apr 2010b; 117 (5): 509-521.

[14] Cho SH, Kim J. Efficacy of acupuncture in management of premenstrual syndrome: A systematic review. Complementary Therapies in Medicine. 2010a; 18 (2): 104-111.

[15] Dhillon S. The acute effect of acupuncture on 20-km cycling performance. Clinical Journal of Sport Medicine. 2008; 18 (1): 7680.

[16] Dorfer L, Moser M, Spindler K, Bahr F, Egarter-Vigl E, Dohr G. 5200-year-old acupuncture in central Europe? Science. Oct 9 1998; 282 (5387): 242-243.

[17] Edwards J, Knowles N. Superficial dry needling and active stretching in the treatment of myofascial pain--a randomised controlled trial. Acupunct Med. 2003; 21 (3): 80-86.

[18] Engberg S, Cohen S, Sereika SM. The efficacy of acupuncture in treating urge and mixed incontinence in women: a pilot study. Journal of Wound, Ostomy, \& Continence Nursing. Nov-Dec 2009; 36 (6): 661-670.

[19] Ernst E, White A. Acupuncture: a scientific appraisal. Oxford: Butterworth-Heinemann; 1999.

[20] Ezzo J, Vickers A, Richardson MA, et al. Acupuncture-point stimulation for chemotherapy-induced nausea and vomiting. Journal of Clinical Oncology. Oct 1 2005; 23 (28): 7188-7198.

[21] Filshie J, White A. Medical acupuncture: a western scientific approach. Edinburgh: Churchill Livingstone; 1998.

[22] Fink M, Wolkenstein E, Karst M, Gehrke A. Acupuncture in chronic epicondylitis: a randomized controlled trial. Rheumatology. Feb 2002; 41 (2): 205-209.

[23] Fleckenstein J, Zaps D, Ruger LJ, et al. Discrepancy between prevalence and perceived effectiveness of treatment methods in myofascial pain syndrome: Results of a cross-sectional, nationwide survey. Bmc Musculoskeletal Disorders. 2010; 11. Figure 2 PC6 acupuncture point

[24] Frost FA, Jessen B, Siggaardandersen J. Control, double-blind comparison of mepivacaine injection versus saline injection for myofascial pain. Lancet. 1980; 1 (8167): 499-501.

[25] Fung KP, Chow OK, So SY. Attenuation of exercise-induced asthma by acupuncture. Lancet. Dec 20-27 1986; 2 (8521-22): 1419-1422.

[26] Furlan AD, van Tulder MW, Cherkin DC, et al. Acupuncture and dry-needling for low back pain. Cochrane Database Syst Rev. 2005 (1): CD001351.

[27] Gao M, Wang MZ, Li KY, He LF. Changes of mu opioid receptor binding sites in rat brain following electroacupuncture. Acupuncture \& Electro-Therapeutics Research. 1997; 22 (3-4): 161-166.

[28] Garvey TA, Marks MR, Wiesel SW. A prospective, randomised, double-blind evaluation of trigger-point injection therapy for lowback pain. Spine. 1989; 14 (9): 962-964.

[29] Gerwin RD, Shannon S, Hong CZ, Hubbard D, Gevirtz R. Interrater reliability in myofascial trigger point examination. Pain. 1997; 69 (1-2): 65-73.

[30] Gonçalves-Nordon D, Gianini RJ, Regina-Azevedo, G. Development and validation of an index for evaluating the quality of acupuncture articles. Rev Fac Cienc Méd Sorocaba. 2012: 14 (2): 59-63.

[31] Gonçalves-Nordon D, Gianini RJ, Regina-Azevedo, G. Development and validation of an index of the adequacy of the control technique for acupuncture studies. Rev Fac Cienc Méd Sorocaba. 2013: 15 (1): 186-191.

[32] Gonçalves-Nordon D. The relation between quality clinical trials and acupuncture efficacy. Rev Fac Cienc Méd Sorocaba. 2013: 15 (2): 6-10.

[33] Guo HF, Tian J, Wang X, Fang Y, Hou Y, Han J. Brain substrates activated by electroacupuncture (EA) of different frequencies (II): Role of Fos/Jun proteins in EA-induced transcription of preproenkephalin and preprodynorphin genes. Brain Res Mol Brain Res. 1996; 43 (1-2): 167-173. 
[34] Haake M, Muller HH, Schade-Brittinger C, et al. German acupuncture trials (GERAC) for chronic low back pain Randomized, multicenter, blinded, parallel-group trial with 3 groups. Archives of Internal Medicine. 2007; 167 (17): 1892-1898.

[35] Haker E, Lundeberg T. Acupuncture treatment in epicondylalgia: a comparative study of two acupuncture techniques. Clinical Journal of Pain. Sep 1990; 6 (3): 221-226.

[36] Hempen C-H, Chow VW. Pocket atlas of acupuncture. Stuttgart ; New York: Thieme; 2006.

[37] Hong CZ. Lidocaine injection versus dry needling to myofascical trigger point - the importance of the local twitch response. American Journal of Physical Medicine \& Rehabilitation. 1994; 73 (4): 256-263.

[38] Huguenin L, Brukner PD, McCrory P, Smith P, Wajswelner H, Bennell K. Effect of dry needling of gluteal muscles on straight leg raise: a randomised, placebo controlled, double blind trial. British Journal of Sports Medicine. 2005; 39 (2): 84-90.

[39] Hui KKS, Liu J, Makris N, et al. Acupuncture modulates the limbic system and subcortical gray structures of the human brain: Evidence from fMRI studies in normal subjects. Human Brain Mapping. 2000; 9 (1): 13-25.

[40] Hui KKS, Liu J, Marina O, et al. The integrated response of the human cerebro-cerebellar and limbic systems to acupuncture stimulation at ST 36 as evidenced by fMRI. Neuroimage. 2005; 27 (3): 479-496.

[41] Jaeger B, Skootsky SA. Double blind controlled study of different myofascial trigger point injection techniques. Pain. 1987 (SUPPL. 4): S292.

[42] Kalauokalani D, Cherkin DC, Sherman KJ, Koepsell TD, Deyo RA. Lessons from a trial of acupuncture and massage for low back pain: patient expectations and treatment effects. (0362-2436).

[43] Kim L-W, Zhu J. Acupuncture for essential hypertension. Alternative Therapies in Health \& Medicine. Mar-Apr 2010; 16 (2): $18-29$.

[44] Langevin HM, Bouffard NA, Badger GJ, Churchill DL, Howe AK. Subcutaneous tissue fibroblast cytoskeletal remodeling induced by acupuncture: Evidence for a mechanotransduction-based mechanism. Journal of Cellular Physiology. 2006; 207 (3): 767774.

[45] Langevin HM, Yandow JA. Relationship of acupuncture points and meridians to connective tissue planes. Anatomical Record. 2002; 269 (6): 257-265.

[46] Langevin HM. Connective tissue: A body-wide signaling network? Medical Hypotheses. 2006; 66 (6): 1074-1077.

[47] Lee A, Done ML. Stimulation of the wrist acupuncture point P6 for preventing postoperative nausea and vomiting. Cochrane Database of Systematic Reviews. 2004 (3): CD003281.

[48] Lee H, Ernst E. Acupuncture for labor pain management: A systematic review. American Journal of Obstetrics and Gynecology. 2004; 191 (5): 1573-1579.

[49] Lee H, Kim SY, Park J, Kim YJ, Park HJ. Acupuncture for Lowering Blood Pressure: Systematic Review and Meta-analysis. American Journal of Hypertension. 2009; 22 (1): 122-128.

[50] Lee H, Park H-J, Park J, et al. Acupuncture application for neurological disorders. Neurological Research. 2007; 29 Suppl 1: S49-54.

[51] Linde K, Streng A, Jurgens S, et al. Acupuncture for patients with migraine - A randomized controlled trial. Jama-Journal of the American Medical Association. 2005; 293 (17): 2118-2125.

[52] Longbottom J. Acupuncture in manual therapy. Edinburgh: Churchill Livingstone; 2010.

[53] Lund I, Lundeberg T. Are minimal, superficial or sham acupuncture procedures acceptable as inert placebo controls? Acupunct Med. 2006; 24 (1): 13-15.

[54] Lundeberg T, Bondesson L, Thomas M. Effect of acupuncture on experimentally induced itch. British Journal of Dermatology. 1987; 117 (6): 771-777.

[55] Macdonald AJR, Macrae KD, Master BR, Rubin AP. Superficial acupuncture in the relief of chronic low-back-pain - A placebocontrolled randomised trial. Annals of the Royal College of Surgeons of England. 1983; 65 (1): 44-46.

[56] MacPherson H, Asghar A. Acupuncture needle sensations associated with De Qi: a classification based on experts' ratings. Journal of Alternative \& Complementary Medicine. Sep 2006; 12 (7): 633-637.

[57] Manheimer E, White A, Berman B, Forys K, Ernst E. Metaanalysis: Acupuncture for low back pain. Annals of Internal Medicine. 2005; 142 (8): 651-663.
[58] Manheimer E, Zhang G, Udoff L, et al. Effects of acupuncture on rates of pregnancy and live birth among women undergoing in vitro fertilisation: systematic review and meta-analysis. British Medical Journal. 2008; 336 (7643): 545-+.

[59] Mann F. Reinventing acupuncture: a new concept of ancient medicine. 2nd ed. Oxford: Butterworth-Heinemann; 2000.

[60] Melchart D, Linde K, Fischer P, et al. Acupuncture for idiopathic headache. Cochrane Database of Systematic Reviews. 2001 (1): CD001218.

[61] Melzack R, Stillwell DM, Fox EJ. Trigger points and acupuncture points for pain - correlations and implications. Pain. 1977; 3 (1): 3-23.

[62] Molsberger AF, Boewing G, Diener HC, et al. Designing an acupuncture study: The nationwide, randomized, controlled, German acupuncture trials on migraine and tension-type headache. Journal of Alternative and Complementary Medicine. 2006; 12 (3): 237-245.

[63] Mukaino Y, Park J, White A, Ernst E. The effectiveness of acupuncture for depression--a systematic review of randomised controlled trials. Acupuncture in Medicine. Jun 2005; 23 (2): 70 76.

[64] National Institute for Health and Clinical E, National Collaborating Centre for Primary C. Low back pain: early management of persistent non-specific low back pain. London: National Institute for Health and Clinical Excellence; 2009.

[65] Nichols AW, Harrigan R. Complementary and alternative medicine usage by intercollegiate athletes. Clinical Journal of Sport Medicine. May 2006; 16 (3): 232-237.

[66] Njoo KH, Vanderdoes E. The occurence and interrater reliability of myofascial trigger points in the quadratus lumborum and gluteus medius - A prospective-study in nonspecific low-back pain patients and controls in general practice. Pain. 1994; 58 (3): 317323.

[67] Ozerkan KN, Bayraktar B, Yucesir I, Cakir B, Yilddiz F. Effectiveness of Omura's ST.36 point (True ST.36) needling on the Wingate anaerobic test results of young soccer players. Acupuncture \& Electro-Therapeutics Research. 2009; 34 (3-4): 205-216.

[68] Pariente J, White P, Frackowiak RSJ, Lewith G. Expectancy and belief modulate the neuronal substrates of pain treated by acupuncture. Neuroimage. 2005; 25 (4): 1161-1167.

[69] Proctor ML, Smith CA, Farquhar CM, Stones RW. Transcutaneous electrical nerve stimulation and acupuncture for primary dysmenorrhoea. Cochrane Database of Systematic Reviews. 2002 (1): CD002123.

[70] Rampes H, Sharples F, Maragh S, Fisher P. Introducing complementary medicine into the medical curriculum. Journal of the Royal Society of Medicine. 1997; 90 (1): 19-22.

[71] Saladin KS, Van Wynsberghe D. Anatomy and physiology: the unity of form and function. 2nd ed. Boston; London: McGraw-Hill; 2001.

[72] Sandberg M, Lundeberg T, Lindberg LG, Gerdle B. Effects of acupuncture on skin and muscle blood flow in healthy subjects. European Journal of Applied Physiology. 2003; 90 (1-2): 114-119.

[73] Sato A, Sato Y, Shimura M, Uchida S. Calcitonin gene-related peptide produces skeletal muscle vasodilation following antidromic stimulation of unmyelinated afferents in the dorsal root in rats. Neuroscience Letters. 2000; 283 (2): 137-140.

[74] Simons DG, Travell JG, Simons LS. Travell and Simons' myofascial pain and dysfunction: The trigger point manual, Vol. 1. Upper half of body, Second edition. Travell and Simons' myofascial pain and dysfunction: The trigger point manual, Vol. 1. Upper half of body, Second edition. 1999: xviii+1038p.

[75] Smidt N, van der Windt DAWM, Assendelft WJJ, Deville WLJM, Korthals-de Bos IBC, Bouter LM. Corticosteroid injections, physiotherapy, or a wait-and-see policy for lateral epicondylitis: a randomised controlled trial.[Summary for patients in Aust J Physiother. 2002; 48 (3): 239; PMID: 12369566]. Lancet. Feb 23 2002; 359 (9307): 657-662.

[76] Smith C, Crowther C, Beilby J. Acupuncture to treat nausea and vomiting in early pregnancy: a randomized controlled trial. Birth. Mar 2002; 29 (1): 1-9.

[77] Smith CA, Hay PP, Macpherson H. Acupuncture for depression. Cochrane Database of Systematic Reviews. 2010 (1): CD004046.

[78] Stener-Victorin E, Humaidan P. Use of acupuncture in female infertility and a summary of recent acupuncture studies related to embryo transfer. Acupunct Med. 2006; 24 (4): 157-163. 
[79] Thomas KJ, MacPherson H, Ratcliffe J, et al. Longer term clinical and economic benefits of offering acupuncture care to patients with chronic low back pain. Health Technology Assessment. 2005; 9 (32): III-+.

[80] Thomas M, Eriksson SV, Lundeberg T. A comparitive-study of diazepam and acupuncture in patients with osteoarthritis pain - A placebo controlled-study. American Journal of Chinese Medicine. 1991; 19 (2): 95-100.

[81] Tough EA, White AR, Cummings TM, Richards SH, Campbell JL. Acupuncture and dry needling in the management of myofascial trigger point pain: A systematic review and meta-analysis of randomised controlled trials. European Journal of Pain. 2009; 13 (1): 3-10.

[82] Treede RD, Meyer RA, Raja SN, Campbell JN. Peripheral and central mechanisms of cutaneous hyperalgesia. Progress in Neurobiology. 1992; 38 (4): 397-421.

[83] Trinh KV, Phillips SD, Ho E, Damsma K. Acupuncture for the alleviation of lateral epicondyle pain: a systematic review. Rheumatology. Sep 2004; 43 (9): 1085-1090.

[84] Vickers AJ, Rees RW, Zollman CE, et al. Acupuncture for chronic headache in primary care: large, pragmatic, randomised trial. BMJ. Mar 27 2004; 328 (7442): 744.

[85] Wall PD, Melzack R, McMahon SB, Koltzenburg M. Wall and Melzack's textbook of pain. 5th ed. Philadelphia: Elsevier/Churchill Livingstone; 2006.

[86] Wang KM, Yao SM, Xian YL, Hou ZL. A study on the receptive field of acupoints and the relationship between characteristics of needling sensation and groups of afferent fibers. Scientia Sinica Series B-Chemical Biological Agricultural Medical \& Earth Sciences. 1985; 28 (9): 963-977.
[87] Wang KM, Yao SM, Xian YL, Hou ZL. A study on the receptivefield of acupoints and the relationship between characteristics of needling sensation and groups of afferent fibres. Scientia Sinica Series B-Chemical Biological Agricultural Medical \& Earth Sciences. 1985; 28 (9): 963-977.

[88] Weidner C, Klede M, Rukwied R, et al. Acute effects of substance $\mathrm{P}$ and calcitonin gene-related peptide in human skin - A microdialysis study. Journal of Investigative Dermatology. 2000; 115 (6): 1015-1020.

[89] White A, Cummings TM, Filshie J. An introduction to Western medical acupuncture. Edinburgh: Churchill Livingstone Elsevier; 2008.

[90] White A, Foster NE, Cummings M, Barlas P. Acupuncture treatment for chronic knee pain: a systematic review. Rheumatology. 2007; 46 (3): 384-390.

[91] White P. A background to acupuncture and its use in chronic painful musculoskeletal conditions. Journal of the Royal Society for the Promotion of Health. Sep 2006; 126 (5): 219-227.

[92] Williamson L, Yudkin P, Livingstone R, Prasad K, Fuller A, Lawrence $M$. Hay fever treatment in general practice: a randomised controlled trial comparing standardised Western acupuncture with Sham acupuncture. Acupuncture in Medicine. May 1, 1996; 1996; 14: 6-10.

[93] World_Health_Organisation. Acupuncture: Review and analysis of reports on controlled clinical trials: WHO Library Cataloguingin-Publication Data; 2003.

[94] Yu DY, Lee SP. Effect of acupuncture on bronchial asthma. Clinical Science \& Molecular Medicine. Nov 1976; 51 (5): 503509. 